\title{
Chemical Reactivity Properties, Drug-Likeness Features and Bioactivity Scores of the Cholecystokinin Peptide Hormone
}

\author{
Norma Flores-Holguín ${ }^{1}$, Juan Frau ${ }^{2}$, Daniel Glossman-Mitnik ${ }^{1}$ \\ ${ }^{1}$ Laboratorio Virtual NANOCOSMOS, Departamento de Medio Ambiente y Energía, Centro de Investigación en Materiales \\ Avanzados, Chihuahua, Mexico \\ ${ }^{2}$ Departament de Química, Universitat de les Illes Balears, Palma de Mallorca, Spain \\ Email: daniel.glossman@cimav.edu.mx
}

How to cite this paper: Flores-Holguín, N., Frau, J. and Glossman-Mitnik, D. (2019) Chemical Reactivity Properties, Drug-Likeness Features and Bioactivity Scores of the Cholecystokinin Peptide Hormone. Computational Molecular Bioscience, 9, 41-47.

https://doi.org/10.4236/cmb.2019.92004

Received: June 4, 2019

Accepted: June 25, 2019

Published: June 28, 2019

Copyright (๑) 2019 by author(s) and Scientific Research Publishing Inc. This work is licensed under the Creative Commons Attribution International License (CC BY 4.0).

http://creativecommons.org/licenses/by/4.0/

cc) (i) Open Access

\begin{abstract}
Five density functionals, CAM-B3LYP, LC- $\omega$ PBE, MN12SX, N12SX and $\omega \mathrm{B} 97 \mathrm{XD}$, in connection with the Def2TZVP basis set were assessed together with the SMD solvation model for the calculation of the molecular and chemical reactivity properties of the Cholecystokinin peptide hormone (CCK-8) in the presence of water. All the chemical reactivity descriptors for the systems were calculated via Conceptual Density Functional Theory (CDFT). The potential bioavailability and druggability as well as the bioactivity scoresfor CCK- 8 were predicted through different methodologies already reported in the literature which have been previously validated during the study of different peptidic systems. The conclusion was that the CCK- 8 peptide will be moderately bioactive regarding all the interactions.
\end{abstract}

\section{Keywords}

Cholecystokinin Peptide Hormone (CCK-8), Conceptual DFT, Chemical Reactivity, Drug-likeness Features, Bioactivity Scores

\section{Introduction}

For the consideration of therapeutic peptides from the point of view of medicine, it is necessary to know their molecular properties and their bioactivity. It is our belief that the bioactivity of these peptides is intimately related to their chemical reactivity from a molecular perspective. For this reason, we consider it essential to study the chemical reactivity of natural products that have the potential to become medicines through the tools provided by Computational Chemistry and Molecular Modeling. Probably the most powerful tool currently availa- 
ble to study the chemical reactivity of molecular systems from the point of view of Computational Chemistry and Molecular Modeling is the Conceptual DFT [1] [2], also called Chemical Reactivity Theory, which uses a series of global and local descriptors allow to predict the interactions between molecules and understand the way in that chemical reactions proceed. According to our previous studies on peptides, the Conceptual DFT allows to obtain reactivity descriptors which are very reliable and of higher quality in comparison with another theory that are based on the analysis of the wave functions instead of the electronic density.

Considering that the knowledge of the chemical reactivity is essential for the development of new medicines, we have decided to study in this work the peptide hormone called Cholecystokinin (CCK-8), which is involved in the control of appetite. CCK-8 also acts as a neurotransmitter in the brain and is thought to be involved in panic attacks [3]. Thus, the objective of this work is to study the chemical reactivity of CCK- 8 using the techniques of the Conceptual DFT, determining its global reactivity properties, that is, of the molecule as a whole. Moreover, during the process of the development of new drugs, there is a need to learn about the drug-like properties of the involved molecular systems [4]. Thus, the descriptors of bioavailability and bioactivity (Bioactivity Scores) will be calculated through different procedures described in the literature [5] [6] trying to relate them with the calculated Conceptual DFT descriptors.

\section{Computational Methodology}

In the same way, as we have proceeded in our recent studies [7]-[14], the computational tasks in this work have been done by considering the popular Gaussian 09 software [15]. Following the conclusions obtained from those studies, five density functionals have been chosen, CAM-B3LYP, LC- $\omega$ PBE, MN12SX [16], N12SX and $\omega \mathrm{B} 97 \mathrm{XD}$, because they can be considered to be well-behaved for our purposes according to our proposed KID (for Koopmans in DFT) criteria [7]-[14]. For the calculation of the electronic properties, several model chemistries have been considered, based on the mentioned density functionals in connection with the Def2TZVP basis set, while a smaller Def2SVP was considered for the prediction of the most stable structures [17] [18]. In order to obtain accurate results, all calculations were performed in the presence of water simulated with the SMD model (Solvation Model based on the Density) [19].

\section{Results and Discussion}

The molecular structure of CCK-8, which graphical sketch is shown in Figure 1, was preoptimized in the gas phase by considering the DFTBA model available in Gaussian 09 and then reoptimized using the five density functionals mentioned in the previous section together with the Def2SVP basis set and the SMD solvent model using water as the solvent. After verifying that each of the structures corresponded to the minimum energy configurations through a frequency calculation analysis, the electronic properties were determined by using the same model 
chemistry but with the Def2TZVP basis set instead of that used for the geometry optimization (Figure 1).

In order to verify the fulfillment of the KID procedure, it is necessary to perform a comparison of the orbital energies with the results obtained by means of the vertical I and A through the $\triangle S C F$ criterium. To this end, the three main descriptors are linked by $\varepsilon_{H}$ with $-I, \varepsilon_{L}$ with $-\mathrm{A}$, and their behavior in describing the HOMO-LUMO gap as $J_{I}=\left|\varepsilon_{H}+E_{g s}(N-1)-E_{g s}(N)\right|$, $J_{A}=\left|\varepsilon_{L}+E_{g s}(N)-E_{g s(N+1)}\right|$, and $J_{H L}=\sqrt{\left(J_{I}^{2}+J_{A}^{2}\right)}$. Another descriptor, $\Delta \mathrm{SL}$, (the difference between the SOMO and the LUMO) was also designed to guide in verifying the accuracy of the approximation [7]-[13]. The results of this analysis are presented in Table 1.

The overall conclusion that can be extracted from the inspection of the results presented in Table 1 is that, in agreement with our previous studies on melanoidins and peptides, the model chemistries involving the MN12SX and N12SX density functionals are the best for verifying our proposed criteria of well-behavior.

By taking into account the KID procedure presented in our previous works together with the finite difference approximation, the global reactivity descriptors can be expressed as:

\begin{tabular}{ccc}
\hline Electronegativity & $\chi=-0.5(I+A) \approx 0.5\left(\varepsilon_{H}+\varepsilon_{L}\right)$ & {$[1][2]$} \\
Global Hardness & $\eta=(I-A) \approx\left(\varepsilon_{L}-\varepsilon_{H}\right)$ & {$[1][2]$} \\
Electrophilicity & $\omega=0.5 \chi^{2} / \eta$ & {$[20]$} \\
Electrodonating Power & $\omega^{-}=(3 I+A)^{2} / 16(I-A)$ & {$[21]$} \\
Electroaccepting Power & $\omega^{+}=(I+3 A)^{2} / 16(I-A)$ & {$[21]$} \\
Net Electrophilicity & $\Delta \omega^{ \pm}=\omega^{+}+\omega^{-}$ & {$[22]$} \\
\hline
\end{tabular}

where $\varepsilon_{H}$ and $\varepsilon_{L}$ are the energies of the HOMO and LUMO, respectively.

The results for the global reactivity descriptors for CCK- 8 based on the values of the HOMO and LUMO energies calculated with the five density functionals are presented in Table 2.

As expected from the molecular structure of this peptide, its electrodonating ability is more important than its electroaccepting character. It can be seen that MN12SX and N12SX density functionals (which verify the KID criteria) give results different than those obtained from the calculation with the other three density functionals.

The molecular properties that are related to the concept of drug-likeness and in particular those associated with the criteria proposed by Lipinski et al. [23] [24] for the prediction of oral bioavailability have been calculated by feeding the corresponding SMILES notations into the MolInspiration software readily available online (Slovensky Grob, Slovak Republic (https://www.molinspiration.com). The results are presented in Table 3. 


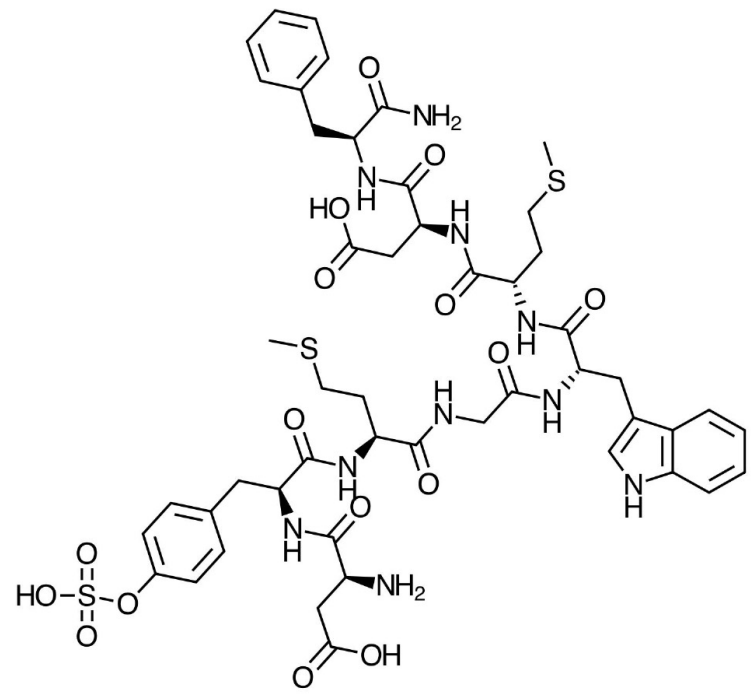

Figure 1. Graphical sketch of the CCK-8 molecule.

Table 1. Total electronic energies of CCK-8 (in au) for the neutral and charged species, the corresponding orbital energies (in eV) and the KID-related descriptors obtained with the five density functionals, the Def2TZVP basis set and water as the solvent.

\begin{tabular}{ccccccccccc}
\hline DF & E0 & E+ & E- & HOMO & LUMO & SOMO & $J_{I}$ & $J_{A}$ & $J_{H L}$ & $\Delta$ SL \\
\hline CAM-B3LYP & -4845.742 & -4845.539 & -4845.772 & -6.923 & 0.611 & -2.246 & 1.391 & 1.416 & 1.985 & 2.857 \\
LC- $\omega$ PBE & -4844.773 & -4844.562 & -4844.510 & -8.243 & 1.474 & -3.489 & 2.484 & 2.465 & 3.500 & 4.963 \\
MN12SX & -4843.970 & -4843.762 & -4944.008 & -5.620 & -1.002 & -1.024 & 0.047 & 0.011 & 0.048 & 0.022 \\
N12SX & -4845.561 & -4845.362 & -4845.593 & -5.390 & -0.824 & -0.903 & 0.017 & 0.041 & 0.045 & 0.079 \\
$\omega$ B97XD & -4846.358 & -4846.152 & -4846.388 & -7.551 & 1.142 & -2.849 & 1.950 & 1.980 & 2.779 & 3.991 \\
\hline
\end{tabular}

Table 2. Global reactivity descriptors (in eV) for CCK-8 calculated with the MN12SX and N12SX density functionals and the Def2TZVP basis set using water as the solvent simulated with the SMD parametrization of the IEF-PCM model.

\begin{tabular}{cccc}
\hline DF & Electronegativity & Global Hardness & Electrophilicity \\
\hline MN12SX & 3.3101 & 4.6170 & 1.1866 \\
N12SX & 3.1067 & 4.5650 & 1.0571 \\
\hline DF & Electrodonating Power & Electroaccepting Power & Net Electrophilicity \\
\hline MN12SX & 2.5383 & 1.2258 & 3.7641 \\
N12SX & 2.3244 & 1.0754 & 3.3998 \\
\hline
\end{tabular}

Table 3. Molecular properties of the CCK-8 peptide calculated to verify the Lipinsky Rule of Five.

\begin{tabular}{cccccccccc}
\hline miLogP & TPSA & nAtoms & nON & nOHNH & nviol & nrotb & volume & MW \\
\hline-4.06 & 4626.80 & 78 & 26 & 15 & 3 & 33 & 974.73 & 1143.29 \\
\hline
\end{tabular}

However, what the Lipinsky Rule of Five really measures is the oral bioavailability of a potential drug because this is desired property for a molecule having drug-like character. Then, a different approach was followed by considering similarity searches in the chemical space of compounds with structures that can be 
compared to those that are being studied and with known pharmacological properties. The same software was used for the calculation of the bioactivity scores which are a measure of the ability of the potential drug to interact with the different receptors, that is, to act as GPCR ligands or Kinase inhibitors, to perform as Ion Channel modulators, or to interact with Enzymes and Nuclear receptors. These predicted bioactivity scores were compared with those resulting of the use of alternative software like MolSoft from Molsoft L.L.C. (San Diego, CA, USA) (http://molsoft.com/mprop/) as well as ChemDoodle Version 9.02 from iChemLabs L.L.C., Richmond, VA, USA (https://www.chemdoodle.com/). The values of the bioactivity scores for CCK- 8 are presented in Table 4 .

These bioactivity scores for organic molecules can be interpreted as active (when the bioactivity score $>0$ ), score lies between -5.0 and 0.0 ) and inactive (when the bioactivity score $<-5.0$ ). Thus, CCK- 8 will be moderately bioactive regarding all the interactions.

\section{Conclusions}

In this paper, we have presented a new study performed on the chemical reactivity of the Cholecystokinin peptide hormone (CCK-8) based on the Conceptual DFT as a tool to explain the molecular interactions.

The knowledge of the values of the global descriptors of the molecular reactivity of CCK- 8 could be useful in the development of new drugs based on this compound or some analogs.

Finally, the molecular properties related to bioavailability and drug-likeness have been predicted using a proven methodology already described in the literature, and the descriptors used for the quantification of the bioactivity allowed to characterize the studied molecule as being moderately bioactive regarding all the interactions with the different receptors.

\section{Acknowledgements}

This work has been partially supported by CIMAV, SC and Consejo Nacional de Ciencia y Tecnología (CONACYT, Mexico) through Grant 219566-2014 for Basic Science Research. Daniel Glossman-Mitnik conducted this work while a Visiting Lecturer at the University of the Balearic Islands from which support is gratefully acknowledged. Norma Flores-Holguín and Daniel Glossman-Mitnik are researchers of CIMAV and CONACYT. This work was cofunded by the Ministerio de Economía y Competitividad (MINECO) and the European Fund for Regional Development (FEDER) (CTQ2014-55835-R).

\section{Conflicts of Interest}

The authors declare no conflicts of interest regarding the publication of this paper.

\section{References}

[1] Parr, R. and Yang, W. (1989) Density Functional Theory of Atoms and Molecules. 
Oxford University Press, New York.

[2] Geerlings, P., De Proft, F. and Langenaeker, W. (2003) Conceptual Density Functional Theory. Chemical Reviews, 103, 1793-1874.

https://doi.org/10.1021/cr990029p

[3] Patrick, G.L. (2013) An Introduction to Medicinal Chemistry. Oxford University Press, Oxford.

[4] Stromgaard, K., Krogsgaard-Larsen, P. and Madsen, U. (2017) Textbook of Drug Design and Discovery. 5th Edition, CRC Press-Taylor \& Francis Group, Boca Raton, Florida.

[5] Gupta, G.K. and Kumar, V. (2016) Drug Design. Walter de Gruyter GmbH, Berlin.

[6] Gore, M. and Jagtap, U.B. (2018) Computational Drug Discovery and Design. Springer, New York. https://doi.org/10.1007/978-1-4939-7756-7

[7] Frau, J. and Glossman-Mitnik, D. (2018) Molecular Reactivity and Absorption Properties of Melanoidin Blue-G1 through Conceptual DFT. Molecules, 23, 559. https://doi.org/10.3390/molecules23030559

[8] Frau, J. and Glossman-Mitnik, D. (2018) Conceptual DFT Study of the Local Chemical Reactivity of the Dilysyldipyrrolones A and B Intermediate Melanoidins. Theoretical Chemistry Accounts, 137, 67. https://doi.org/10.1007/s00214-018-2244-x

[9] Frau, J. and Glossman-Mitnik, D. (2018) Conceptual DFT Study of the Local Chemical Reactivity of the Colored BISARG Melanoidin and Its Protonated Derivative. Frontiers in Chemistry, 6, 1-9. https://doi.org/10.3389/fchem.2018.00136

[10] Frau, J. and Glossman-Mitnik, D. (2018) Molecular Reactivity of Some Maillard Reaction Products Studied through Conceptual DFT. Contemporary Chemistry, 1, $1-14$.

[11] Frau, J. and Glossman-Mitnik, D. (2018) Computational Study of the Chemical Reactivity of the Blue-M1 Intermediate Melanoidin. Computational and Theoretical Chemistry, 1134, 22-29. https://doi.org/10.1016/j.comptc.2018.04.018

[12] Frau, J. and Glossman-Mitnik, D. (2018) Chemical Reactivity Theory Applied to the Calculation of the Local Reactivity Descriptors of a Colored Maillard Reaction Product. Chemical Science International Journal, 22, 1-14. https://doi.org/10.9734/CSJI/2018/41452

[13] Frau, J. and Glossman-Mitnik, D. (2018) Blue M2: An Intermediate Melanoidin Studied via Conceptual DFT. Journal of Molecular Modeling, 24, 138. https://doi.org/10.1007/s00894-018-3673-0

[14] Frau, J., Flores-Holguín, N. and Glossman-Mitnik, D. (2018) Chemical Reactivity Properties, $\mathrm{pKa}$ Values, AGEs Inhibitor Abilities and Bioactivity Scores of the Mirabamides A - H Peptides of Marine Origin Studied by Means of Conceptual DFT. Marine Drugs, 16, 302-319. https://doi.org/10.3390/md16090302

[15] Frisch, M.J., Trucks, G.W., Schlegel, H.B, Scuseria, G.E., Robb, M.A., Cheeseman, J.R., Scalmani, G., Barone, V., Mennucci, B., Petersson, G.A., Nakatsuji, H., Caricato, M., Li, X., Hratchian, H.P., Izmaylov, A.F., Bloino, J., Zheng, G., Sonnenberg, J.L., Hada, M., Ehara, M., Toyota, K., Fukuda, R., Hasegawa, J., Ishida, M., Nakajima, T., Honda, Y., Kitao, O., Nakai, H., Vreven, T., Montgomery Jr., J.A., Peralta, J.E., Ogliaro, F., Bearpark, M., Heyd, J.J., Brothers, E., Kudin, K.N., Staroverov, V.N., Kobayashi, R., Normand, J., Raghavachari, K., Rendell, A., Burant, J.C., Iyengar, S.S., Tomasi, J., Cossi, M., Rega, N., Millam, J.M., Klene, M., Knox, J.E., Cross, J.B., Bakken, V., Adamo, C., Jaramillo, J., Gomperts, R., Stratmann, R.E., Yazyev, O., Austin, A.J., Cammi, R., Pomelli, C., Ochterski, J.W., Martin, R.L., 
Morokuma, K., Zakrzewski, V.G., Voth, G.A., Salvador, P., Dannenberg, J.J., Dapprich, S., Daniels, A.D., Farkas, O., Foresman, J.B., Ortiz, J.V., Cioslowski, J. and Fox, D.J. (2016) Gaussian 09 Revision E.01. Gaussian Inc., Wallingford CT.

[16] Peverati, R. and Truhlar, D.G. (2012) Screened-Exchange Density Functionals with Broad Accuracy for Chemistry and Solid-State Physics. Physical Chemistry Chemical Physics, 14, 16187-16191. https://doi.org/10.1039/c2cp42576a

[17] Weigend, F. and Ahlrichs, R. (2005) Balanced Basis Sets of Split Valence, Triple Zeta Valence and Quadruple Zeta Valence Quality for H to Rn: Design and Assessment of Accuracy. Physical Chemistry Chemical Physics, 7, 3297-3305. https://doi.org/10.1039/b508541a

[18] Weigend, F. (2006) Accurate Coulomb-Fitting Basis Sets for $\mathrm{H}$ to Rn. Physical Chemistry Chemical Physics, 8, 1057-1065. https://doi.org/10.1039/b515623h

[19] Marenich, A., Cramer, C. and Truhlar, D. (2009) Universal Solvation Model Based on Solute Electron Density and a Continuum Model of the Solvent Defined by the Bulk Dielectric Constant and Atomic Surface Tensions. Journal of Physical Chemistry B, 113, 6378-6396. https://doi.org/10.1021/jp810292n

[20] Parr, R., Szentpaly, L. and Liu, S. (1999) Electrophilicity Index. Journal of the American Chemical Society, 121, 1922-1924. https://doi.org/10.1021/ja983494x

[21] Gázquez, J., Cedillo, A. and Vela, A. (2007) Electrodonating and Electroaccepting Powers. Journal of Physical Chemistry A, 111, 1966-1970. https://doi.org/10.1021/jp065459f

[22] Chattaraj, P., Chakraborty, A. and Giri, S. (2009) Net Electrophilicity. Journal of Physical Chemistry A, 113, 10068-10074. https://doi.org/10.1021/jp904674x

[23] Lipinski, C., Lombardo, F., Dominy, B. and Feeney, P. (2001) Experimental and Computational Approaches to Estimate Solubility and Permeability in Drug Discovery and Development Settings. Advanced Drug Delivery Reviews, 46, 3-26. https://doi.org/10.1016/S0169-409X(00)00129-0

[24] Leeson, P. (2012) Drug Discovery: Chemical Beauty Contest. Nature, 481, 455-456. https://doi.org/10.1038/481455a 